\title{
An Expanded Mixed Finite Element Method for Space Fractional Darcy Flow in Porous Media*
}

\author{
Huangxin Chen ${ }^{1}$ and Shuyu Sun ${ }^{2}$ \\ 1 School of Mathematical Sciences and Fujian Provincial Key Laboratory on \\ Mathematical Modeling and High Performance Scientific Computing, Xiamen \\ University, Fujian, 361005, China, and King Abdullah University of Science and \\ Technology (KAUST), Physical Science and Engineering Division (PSE), Thuwal, \\ 23955-6900, Saudi Arabia. \\ chx@xmu.edu.cn \\ 2 King Abdullah University of Science and Technology (KAUST), Physical Science \\ and Engineering Division (PSE), Thuwal, 23955-6900, Saudi Arabia. \\ shuyu. sun@kaust.edu.sa
}

\begin{abstract}
In this paper an expanded mixed formulation is introduced to solve the two dimensional space fractional Darcy flow in porous media. By introducing an auxiliary vector, we derive a new mixed formulation and the well-possedness of the formulation can be established. Then the locally mass-conservative expanded mixed finite element method is applied for the solution. Numerical results are shown to verify the efficiency of the proposed algorithm.
\end{abstract}

Keywords: Space fractional Darcy flow · Extended mixed finite element method · Well-posedness.

\section{Introduction}

Fractional partial differential equations (PDE) have been explored as an important tool to develop more accurate mathematical models to describe complex anomalous systems such as phase transitions, anomalous diffusions. In this paper, we focus on the modeling and simulation of flow in porous media. In particular, when considering modeling of flow in fractured porous media, one may consider different fractional time derivatives in matrix and fracture regions due to the different memory properties. For instance, Caputo [3] apply the time fractional PDE to model the flow in fractured porous media. However, there still exists the fact that there is a steady state for flow in fractured porous media. Thus, we consider the steady state space fractional PDE for the modeling and simulation of flow in porous media.

* The work of Huangxin Chen was supported by the NSF of China (Grant No. 11771363, 91630204, 51661135011), the Fundamental Research Funds for the Central Universities (Grant No. 20720180003). The work of Shuyu Sun was supported by King Abdullah University of Science and Technology (KAUST) through the grant BAS/1/1351-01-01. Shuyu Sun is the corresponding author. 
Until now, a number of articles referring to the space fractional PDE have appeared in literature (see [13] and the reference therein). Most of the works concern on the fractional Laplacian equation in unbounded or bounded domain, and there exist different kinds of definitions of fractional Laplacian, such as spectral/Fourier definition, singular integral representation, via the standard Laplacian (elliptic extension), directional representation, et al. Lots of numerical methods have been developed for the space fractional PDE, for instance, the finite different method, the finite volume method, the spectral method, et al (see $[9,12,15,16,19,14]$ and the references therein). In particular, the finite element methods have been firstly developed and analyzed by Ervin, Roop for the space fractional PDE, and then by other authors in a series of works $[5,18,6,7,2]$. In these works, standard Galerkin finite element methods are always applied for the fractional Laplacian equation. However, when the standard Galerkin finite element methods are applied to the flow equation, the mass conservation can not be retained.

For the space fractional Darcy flow, J. H. He [10] firstly studied seepage flow in porous media and used fractional derivatives to describe the fractional Darcy's

law behavior. In [10], the permeability can only be assumed to be diagonal and the PDE system violates the principle of Galilean invariance. Some numerical methods have been developed for such kind of equation, e.g., see [2]. In this paper, we will apply the fractional gradient operator defined by Meerschaert et al [17] to write down the space fractional Darcy flow for the two dimensional problem which obeys the principle of Galilean invariance. In order to develop the locally mass-conservative finite element method for the fractional Darcy flow, Chen and Wang [4] proposed a new mixed finite element method for a one-dimensional fractional Darcy flow. In this work, we extend the locally massconservative mixed finite element method to the two dimensional problems which can be easily extended to three dimensional problems. By introducing a new auxiliary vector, we can obtain the new expanded mixed formulation for the fractional Darcy flow and the well-possedness of the new formulation can be well established.

The rest of the paper is organized as follows. In Section 2, we introduce the mathematical model for the two dimensional space fractional Darcy flow in porous media. Then we introduce the expanded mixed formulation and establish its well-posedness in Section 3, and show the expanded mixed finite element method and the detailed implementation in Section 4. Some numerical results are given in Section 5 to verify the efficiency of the proposed algorithm. Finally we provide a conclusion in Section 6 .

\section{Preliminary}

In this section we will follow $[7,17]$ to recall the definitions of the directional integral, the directional derivative operators, the fractional gradient operator, and then introduce the space fractional Darcy's law which obeys the principle of Galilean invariance. We use the standard notations and definitions for Sobolev 
spaces (cf. [1]) throughout the paper. Since our work focus on the two dimensional problem, the following definitions are given for the functions in $\mathcal{R}^{2}$.

Definition 1. (cf. [7]) Let $\mu>0$ and $\theta \in \mathcal{R}$. The $\mu$-th order fractional integral in the direction $\boldsymbol{\theta}=(\cos \theta, \sin \theta)$ is defined by

$$
D_{\theta}^{-\mu} v(x, y)=\int_{0}^{\infty} \frac{\xi^{\mu-1}}{\Gamma(\xi)} v(x-\xi \cos \theta, y-\xi \sin \theta) d \xi
$$

where $\Gamma(\cdot, \cdot)$ is a Gamma function.

Definition 2. (cf. [7]) Let $n$ be a positive integer. The $n$-th order derivative in the direction of $\boldsymbol{\theta}=(\cos \theta, \sin \theta)$ is given by

$$
D_{\theta}^{n} v(x, y)=\left(\cos \theta \frac{\partial}{\partial x}+\sin \theta \frac{\partial}{\partial y}\right)^{n} v(x, y)
$$

Definition 3. (cf. [7]) Let $\mu>0, \theta \in \mathcal{R}$. Let $n$ be an integer such that $n-1 \leq$ $\mu<n$, and define $\sigma=n-\mu$. Then the $\mu$-th order directional derivative in the direction of $\boldsymbol{\theta}=(\cos \theta, \sin \theta)$ is defined by

$$
D_{\theta}^{\mu} v(x, y)=D_{\theta}^{-\sigma} D_{\theta}^{n} v(x, y)
$$

Definition 4. (cf. [17]) Let $\alpha \in(0,1)$. The fractional gradient operator with respect to the measure $M$ is defined by

$$
\nabla_{M}^{\alpha} v=\int_{0}^{2 \pi} \boldsymbol{\theta} D_{\theta}^{\alpha} v M(\theta) d \theta
$$

where $\boldsymbol{\theta}=(\cos \theta, \sin \theta)$ is a unit vector, $D_{\theta}^{\alpha}$ is the Riemann-Liouville fractional directional derivative and $M(\theta)$ is a positive (probability) density function satisfying $\int_{0}^{2 \pi} M(\theta) d \theta=1$.

By the Lemma 5.6 in [7], we have $D_{\theta}^{\alpha} v=D_{\theta}^{\alpha-1} D_{\theta}^{1} v=D_{\theta}^{\alpha-1}(\boldsymbol{\theta} \cdot \nabla v)$. Thus, we write down the steady state space fractional Darcy flow as follows:

$$
\begin{aligned}
\nabla \cdot \boldsymbol{u} & =f & & \text { in } \quad \Omega, \\
\boldsymbol{u} & =-\boldsymbol{K} \nabla_{M}^{\alpha} p & & \text { in } \quad \Omega, \\
p & =0 & & \text { in } \quad \mathcal{R}^{2} \backslash \Omega,
\end{aligned}
$$

where $\boldsymbol{u}, p, f$ are fluid velocity, pressure and source term, $\boldsymbol{K}$ is a bounded symmetric and positive definite permeability tensor, and $\nabla_{M}^{\alpha} p=\int_{0}^{2 \pi} \boldsymbol{\theta} D_{\theta}^{\alpha-1}(\boldsymbol{\theta}$. $\nabla p) M(\theta) d \theta$. For brevity, we consider the fractional Darcy flow in a bounded domain with homogeneous boundary condition for pressure and assume the pressure to be zero outside the domain. For the problem with non-homogeneous boundary conditions, some addition techniques such as the lifting approach and other strategies introduced in [13] can be further applied to solve the problem. 
For the one dimensional space fractional diffusion equation, the regularity of solution was obtained in $[11,8]$. In this work, we consider the two dimensional model and assume the regularity of solution for the system $(2.1)$ as $p \in H_{0}^{\frac{\alpha+1}{2}}(\Omega)$. The gravity effect can also be considered in the space fractional Darcy law as $\boldsymbol{u}=-\boldsymbol{K}\left(\nabla_{M}^{\alpha} p+\rho \boldsymbol{g}\right)$, and the above model can also be extended to the three dimensional problem.

\section{Expanded mixed formulation}

In this section we present a mass-conservative mixed formulation for the fractional Darcy flow (2.1) and establish the well-posedness of the weak formulation. Firstly we define the notations for some Sobolev spaces as follows:

$$
\boldsymbol{V}:=H(\operatorname{div}, \Omega) \cap\left[H^{\frac{1-\alpha}{2}}(\Omega)\right]^{2}, \quad \boldsymbol{H}:=\left[H^{\frac{\alpha-1}{2}}(\Omega)\right]^{2}, \quad Q:=L^{2}(\Omega) .
$$

In order to propose a well-posed mixed formulation for the space fractional Darcy flow, we introduce a new auxiliary vector $\boldsymbol{w}=\nabla p \in \boldsymbol{H}$. Now we present the expanded mixed formulation for (2.1) as follows: Find $(\boldsymbol{u}, \boldsymbol{w}, p) \in \boldsymbol{V} \times \boldsymbol{H} \times Q$, such that

$$
\begin{aligned}
(\boldsymbol{w}, \boldsymbol{\eta})+(p, \nabla \cdot \boldsymbol{\eta}) & =0, \\
(\boldsymbol{u}, \boldsymbol{v})+\left(\boldsymbol{K} \int_{0}^{2 \pi} \boldsymbol{\theta} D_{\theta}^{\alpha-1}(\boldsymbol{\theta} \cdot \boldsymbol{w}) M(\theta) d \theta, \boldsymbol{v}\right) & =0, \\
(\nabla \cdot \boldsymbol{u}, q) & =(f, q),
\end{aligned}
$$

for any $(\boldsymbol{\eta}, \boldsymbol{v}, q) \in \boldsymbol{V} \times \boldsymbol{H} \times Q$. Since we assume that $\boldsymbol{V}$ and $\boldsymbol{H}$ are dual spaces, $\boldsymbol{w}, \boldsymbol{u} \in \boldsymbol{V}$ and $\boldsymbol{\eta}, \boldsymbol{v} \in \boldsymbol{H}$, we can see that the inner products for $(\boldsymbol{w}, \boldsymbol{\eta})$ and $(\boldsymbol{u}, \boldsymbol{v})$ in (3.1) are well defined.

Now we define $\boldsymbol{U}:=\boldsymbol{H} \times Q$ and let

$$
\begin{aligned}
a(\boldsymbol{\tau}, \boldsymbol{\chi}) & :=\left(\boldsymbol{K} \int_{0}^{2 \pi} \boldsymbol{\theta} D_{\theta}^{\alpha-1}(\boldsymbol{\theta} \cdot \boldsymbol{w}) M(\theta) d \theta, \boldsymbol{v}\right) \\
b(\boldsymbol{\chi}, \boldsymbol{u}) & :=(\boldsymbol{u}, \boldsymbol{v})+(\nabla \cdot \boldsymbol{u}, q) \\
b(\boldsymbol{\tau}, \boldsymbol{\eta}) & :=(\boldsymbol{w}, \boldsymbol{\eta})+(p, \nabla \cdot \boldsymbol{\eta})
\end{aligned}
$$

for $\boldsymbol{\tau}=(\boldsymbol{w}, p) \in \boldsymbol{U}, \boldsymbol{\chi}=(\boldsymbol{v}, q) \in \boldsymbol{U}$. Then the expanded mixed formulation (3.1) can be equivalently rewritten as follows: For any $(\boldsymbol{\chi}, \boldsymbol{\eta}) \in \boldsymbol{U} \times \boldsymbol{V}$, find $(\boldsymbol{\tau}, \boldsymbol{u}) \in \boldsymbol{U} \times \boldsymbol{V}$ such that

$$
\begin{aligned}
a(\boldsymbol{\tau}, \boldsymbol{\chi})+b(\boldsymbol{\chi}, \boldsymbol{u}) & =(f, q), \\
b(\boldsymbol{\tau}, \boldsymbol{\eta}) & =0 .
\end{aligned}
$$

In the following, we will aim to prove the well-posedness of the mixed system (3.2). We define $\boldsymbol{Z}=\{\boldsymbol{\chi} \in \boldsymbol{U}: b(\boldsymbol{\chi}, \boldsymbol{\eta})=0, \forall \boldsymbol{\eta} \in \boldsymbol{V}\}$. We start the proof from the following key lemma which can be proved by the similar technique in [4]. We

denote by $C$ with or without subscript a positive constant. These constants can take on different values in different occurrences. 
Lemma 1. Let $\boldsymbol{\chi}=(\boldsymbol{v}, q) \in \boldsymbol{Z}$. We have that $\boldsymbol{\chi} \in \boldsymbol{Z}$ if and only if $q \in H_{0}^{\frac{\alpha+1}{2}}(\Omega)$ and $\boldsymbol{v}=\nabla q \in\left[H^{\frac{\alpha-1}{2}}(\Omega)\right]^{2}$.

Proof. If $q \in H_{0}^{\frac{\alpha+1}{2}}(\Omega)$ and $\boldsymbol{v}=\nabla q \in\left[H^{\frac{\alpha-1}{2}}(\Omega)\right]^{2}$, we have

$$
(\boldsymbol{v}, \boldsymbol{\eta})=(\nabla q, \boldsymbol{\eta})=-(q, \nabla \cdot \boldsymbol{\eta})+\int_{\partial \Omega} q \boldsymbol{\eta} \cdot \boldsymbol{n}, \quad \forall \boldsymbol{\eta} \in \boldsymbol{V} .
$$

Since $q \in H_{0}^{\frac{\alpha+1}{2}}(\Omega)$, we have $(\boldsymbol{v}, \boldsymbol{\eta})+(q, \nabla \cdot \boldsymbol{\eta})=0$, i.e., $\boldsymbol{\chi}=(\boldsymbol{v}, q) \in \boldsymbol{Z}$.

Now we let $\boldsymbol{\chi}=(\boldsymbol{v}, q) \in \boldsymbol{Z}$. Firstly, we let $\boldsymbol{\eta}=(1,0)^{T}$ or $(0,1)^{T}$ in $b(\boldsymbol{\chi}, \boldsymbol{\eta})$ and we get $\boldsymbol{v} \in\left[L^{1}(\Omega)\right]^{2}$. Then we let $\boldsymbol{\eta} \in \mathcal{D}(\Omega)$ where $\mathcal{D}(\Omega)$ denotes the set of all functions $\phi \in C^{\infty}(\Omega)$ which vanish outside a compact subset of $\Omega$. Then we have

$$
(\boldsymbol{v}, \boldsymbol{\eta})=-(q, \nabla \cdot \boldsymbol{\eta})=(\nabla q, \boldsymbol{\eta}), \quad \forall \boldsymbol{\eta} \in[\mathcal{D}(\Omega)]^{2},
$$

which yields that $\boldsymbol{v}=\nabla q \in\left[L^{1}(\Omega)\right]^{2}$ and $q \in W^{1,1}(\Omega)$. By the density and Sobolev imbedding theories, we have that for any $q \in W^{1,1}(\Omega)$, there exist $q_{\epsilon} \in C^{1}(\Omega)$ and a constant $C>0$ such that

$$
\left\|q-q_{\epsilon}\right\|_{W^{1,1}(\Omega)} \leq \epsilon, \quad\left\|q-q_{\epsilon}\right\|_{L^{\infty}(\Omega)} \leq C\left\|q-q_{\epsilon}\right\|_{W^{1,1}(\Omega)} \leq C \epsilon .
$$

Now, for any $\boldsymbol{\eta} \in \boldsymbol{V}$, we have

$$
\begin{aligned}
(q, \nabla \cdot \boldsymbol{\eta})= & \left(q-q_{\epsilon}, \nabla \cdot \boldsymbol{\eta}\right)+\left(q_{\epsilon}, \nabla \cdot \boldsymbol{\eta}\right) \\
= & \left(q-q_{\epsilon}, \nabla \cdot \boldsymbol{\eta}\right)-\left(\nabla q_{\epsilon}, \boldsymbol{\eta}\right)+\int_{\partial \Omega} q_{\epsilon} \boldsymbol{\eta} \cdot \boldsymbol{n} \\
= & -(\nabla q, \boldsymbol{\eta})+\int_{\partial \Omega} q \boldsymbol{\eta} \cdot \boldsymbol{n} \\
& +\left(q-q_{\epsilon}, \nabla \cdot \boldsymbol{\eta}\right)-\left(\nabla q_{\epsilon}-\nabla q, \boldsymbol{\eta}\right)+\int_{\partial \Omega}\left(q_{\epsilon}-q\right) \boldsymbol{\eta} \cdot \boldsymbol{n} .
\end{aligned}
$$

By the density argument and the imbedding theory, we can see that the last three terms on the right-hand side of the last equality in (3.3) become zero as $\epsilon \rightarrow 0$. Thus combining the above derivation and $\boldsymbol{v}=\nabla q$, we have

$$
(q, \nabla \cdot \boldsymbol{\eta})=-(\nabla q, \boldsymbol{\eta})+\int_{\partial \Omega} q \boldsymbol{\eta} \cdot \boldsymbol{n}=-(\boldsymbol{v}, \boldsymbol{\eta})+\int_{\partial \Omega} q \boldsymbol{\eta} \cdot \boldsymbol{n}, \quad \forall \boldsymbol{\eta} \in \boldsymbol{V} .
$$

Since $\boldsymbol{\chi}=(\boldsymbol{v}, q) \in \boldsymbol{Z}$, we have $(q, \nabla \cdot \boldsymbol{\eta})+(\boldsymbol{v}, \boldsymbol{\eta})=0$, which together with (3.4) yields that $q=0$ on $\partial \Omega$. Thus we have $q \in W_{0}^{1,1}(\Omega)$.

By the definition of fractional gradient operator, we have

$$
\begin{aligned}
\nabla_{M}^{\frac{\alpha+1}{2}} q=\int_{0}^{2 \pi} \boldsymbol{\theta} D_{\theta}^{\frac{\alpha+1}{2}} q M(\theta) d \theta & =\int_{0}^{2 \pi} \boldsymbol{\theta} D_{\theta}^{\frac{\alpha-1}{2}} \boldsymbol{\theta} \cdot \nabla q M(\theta) d \theta \\
& =\int_{0}^{2 \pi} \boldsymbol{\theta} D_{\theta}^{\frac{\alpha-1}{2}} \boldsymbol{\theta} \cdot \boldsymbol{v} M(\theta) d \theta \in L^{2}(\Omega)
\end{aligned}
$$

Thus we obtain $q \in H^{\frac{\alpha+1}{2}}(\Omega)$ which together with $q=0$ on $\partial \Omega$ yields $q \in$ $H_{0}^{\frac{\alpha+1}{2}}(\Omega)$ and $\boldsymbol{v}=\nabla q \in\left[H^{\frac{\alpha-1}{2}}(\Omega)\right]^{2}$. Now we conclude the proof. 
Next we introduce another two important tools to prove the well-posedness of $(3.2)$.

Lemma 2. (cf. [y]) Let $\mu>0$. For each $\theta \in[0,2 \pi)$, there holds

$\left(D_{\theta}^{\mu} q, D_{\theta+\pi}^{\mu} q\right)_{L^{2}(\Omega)}=\cos (\pi \mu)\left\|D_{\theta}^{\mu} q\right\|_{L^{2}(\Omega)}^{2}, \quad \forall q \in H_{0}^{\mu}(\Omega)$ and $q=0$ in $\mathcal{R}^{2} \backslash \Omega$.

Lemma 3. (cf. [7]) For any $q \in H_{0}^{\mu}(\Omega)$ and $q=0$ in $\mathcal{R}^{2} \backslash \Omega$, there holds the fractional Poincaré-Friedrichs inequality as follows:

$$
\int_{0}^{2 \pi}\left\|D_{\theta}^{\mu} q\right\|_{L^{2}(\Omega)}^{2} M(\theta) d \theta \geq C\|q\|_{L^{2}(\Omega)}^{2} .
$$

Now for any $\boldsymbol{\chi}=(\boldsymbol{v}, q) \in \boldsymbol{U}$, we denote

$$
|\boldsymbol{v}|_{\frac{\alpha-1}{2}, M}^{2}=\int_{0}^{2 \pi}\left\|D_{\theta}^{\frac{\alpha-1}{2}} \boldsymbol{\theta} \cdot \boldsymbol{v}\right\|_{L^{2}(\Omega)}^{2} M(\theta) d \theta
$$

and

$$
\|\chi\|_{\boldsymbol{U}}^{2}:=\|q\|_{L^{2}(\Omega)}^{2}+|\boldsymbol{v}|_{\frac{\alpha-1}{2}, M}^{2} .
$$

In the following, in order to show the proof in brevity, we assume the permeability tensor $\boldsymbol{K}=K \boldsymbol{I}$ with a positive constant $K>0$ and $\boldsymbol{I}$ is identity matrix.

Lemma 4. For any $\chi \in Z$, we have

$$
a(\chi, \chi) \geq C\|\chi\|_{U}^{2}
$$

Proof. By Lemma 1, for any $\boldsymbol{\chi}=(\boldsymbol{v}, q) \in \boldsymbol{Z}$, we have $\boldsymbol{v}=\nabla q$ and $q \in H_{0}^{\frac{\alpha+1}{2}}(\Omega)$. Then by the Theorems 2.1-2.2 in [7], the fact $D_{\theta}^{1}=-D_{\theta+\pi}^{1}$ and the Lemma 2, we have

$$
\begin{aligned}
a(\boldsymbol{\chi}, \boldsymbol{\chi}) & =\left(K \int_{0}^{2 \pi} \boldsymbol{\theta} D_{\theta}^{\alpha-1}(\boldsymbol{\theta} \cdot \boldsymbol{v}) M(\theta) d \theta, \boldsymbol{v}\right) \\
& =\left(K \int_{0}^{2 \pi} D_{\theta}^{\alpha-1}(\boldsymbol{\theta} \cdot \nabla q) M(\theta) d \theta, \boldsymbol{\theta} \cdot \nabla q\right) \\
& =\int_{0}^{2 \pi}\left(K D_{\theta}^{\frac{\alpha-1}{2}}(\boldsymbol{\theta} \cdot \nabla q), D_{\theta+\pi}^{\frac{\alpha-1}{2}}(\boldsymbol{\theta} \cdot \nabla q)\right) M(\theta) d \theta \\
& =-\int_{0}^{2 \pi}\left(K D_{\theta}^{\frac{\alpha+1}{2}} q, D_{\theta+\pi}^{\frac{\alpha+1}{2}} q\right) M(\theta) d \theta \\
& =\sin \frac{\pi \alpha}{2} \int_{0}^{2 \pi}\left\|\sqrt{K} D_{\theta}^{\frac{\alpha+1}{2}} q\right\|_{L^{2}(\Omega)}^{2} M(\theta) d \theta
\end{aligned}
$$

We note that $D_{\theta}^{\frac{\alpha+1}{2}} q=D_{\theta}^{\frac{\alpha-1}{2}} \boldsymbol{\theta} \cdot \boldsymbol{v}$. Then we can get the desired estimate by the above equality and the fractional Poincaré-Friedrichs inequality in the Lemma 3. 
Lemma 5. There holds

$$
\inf _{\boldsymbol{\eta} \in \boldsymbol{V}} \sup _{\boldsymbol{\chi} \in \boldsymbol{U}} \frac{b(\boldsymbol{\chi}, \boldsymbol{\eta})}{\|\boldsymbol{\chi}\|_{\boldsymbol{U}}\|\boldsymbol{\eta}\|_{\boldsymbol{V}}} \geq C
$$

Proof. For any $\boldsymbol{\eta} \in \boldsymbol{V}$, we let $\boldsymbol{\chi}_{0}=(\boldsymbol{\eta}, \nabla \cdot \boldsymbol{\eta})$, then we have

$$
\sup _{\boldsymbol{\chi} \in \boldsymbol{U}} \frac{b(\boldsymbol{\chi}, \boldsymbol{\eta})}{\|\boldsymbol{\chi}\|_{\boldsymbol{U}}} \geq \frac{b\left(\boldsymbol{\chi}_{0}, \boldsymbol{\eta}\right)}{\left\|\boldsymbol{\chi}_{0}\right\|_{\boldsymbol{U}}}=\frac{(\boldsymbol{\eta}, \boldsymbol{\eta})+(\nabla \cdot \boldsymbol{\eta}, \nabla \cdot \boldsymbol{\eta})}{\left(\|\boldsymbol{\eta}\|_{\frac{\alpha-1}{2}, M}^{2}+\|\nabla \cdot \boldsymbol{\eta}\|_{L^{2}(\Omega)}^{2}\right)^{1 / 2}} .
$$

By the imbedding theory, we easily have

$$
\sup _{\boldsymbol{\chi} \in \boldsymbol{U}} \frac{b(\boldsymbol{\chi}, \boldsymbol{\eta})}{\|\boldsymbol{\chi}\|_{\boldsymbol{U}}} \geq C\|\boldsymbol{\eta}\|_{\boldsymbol{V}}
$$

which directly yields the desired estimate.

By the Lemmas 4-5 and the Babuška-Brezzi theory, we finally obtain the following theorem to state the well-posedness of (3.2), and this also indicates the well-posedness of the mixed system (3.1).

Theorem 1. There exists a unique solution $(\boldsymbol{\tau}, \boldsymbol{u})$ for the mixed system (3.2).

\section{Expanded mixed finite element method and its implementation}

In this section we will introduce the expanded mixed finite element method for the mixed formulation (3.1) and show the details of implementation. Let $\mathcal{T}_{h}$ be the quasi-uniform structured or unstructured mesh on $\Omega$. We define

$$
\begin{aligned}
\boldsymbol{V}_{h} & =\left\{\boldsymbol{\eta}_{h} \in \boldsymbol{V}:\left.\boldsymbol{\eta}_{h}\right|_{T} \in R T_{0}(T), \forall T \in \mathcal{T}_{h}\right\}, \\
\boldsymbol{H}_{h} & =\left\{\boldsymbol{v}_{h} \in \boldsymbol{H}:\left.\boldsymbol{v}_{h}\right|_{T} \in\left[P_{0}(T)\right]^{2}, \forall T \in \mathcal{T}_{h}\right\}, \\
Q_{h} & =\left\{q_{h} \in Q:\left.q_{h}\right|_{T} \in P_{0}(T), \forall T \in \mathcal{T}_{h}\right\} .
\end{aligned}
$$

We remark that the high order mixed finite element spaces can also be used in $\boldsymbol{V}_{h} \times \boldsymbol{H}_{h} \times Q_{h}$ if the solution is smooth enough. For the problem with a low regularity solution, the approximation based on the low order mixed finite element space is advised. We utilize $\boldsymbol{V}_{h}, \boldsymbol{H}_{h}, Q_{h}$ as the approximate spaces for $\boldsymbol{V}, \boldsymbol{H}, Q$. The expanded mixed finite element method for the space fractional Darcy flow (2.1) is defined as: Find $\left(\boldsymbol{u}_{h}, \boldsymbol{w}_{h}, p_{h}\right) \in \boldsymbol{V}_{h} \times \boldsymbol{H}_{h} \times Q_{h}$ such that

$$
\begin{aligned}
\left(\boldsymbol{w}_{h}, \boldsymbol{\eta}_{h}\right)+\left(p_{h}, \nabla \cdot \boldsymbol{\eta}_{h}\right) & =0, \\
\left(\boldsymbol{u}_{h}, \boldsymbol{v}_{h}\right)+\left(\boldsymbol{K} \int_{0}^{2 \pi} \boldsymbol{\theta} D_{\theta}^{\alpha-1}\left(\boldsymbol{\theta} \cdot \boldsymbol{w}_{h}\right) M(\theta) d \theta, \boldsymbol{v}_{h}\right) & =0, \\
\left(\nabla \cdot \boldsymbol{u}_{h}, q_{h}\right) & =\left(f, q_{h}\right),
\end{aligned}
$$


for any $\left(\boldsymbol{\eta}_{h}, \boldsymbol{v}_{h}, q_{h}\right) \in \boldsymbol{V}_{h} \times \boldsymbol{H}_{h} \times Q_{h}$.

We note that the key step in implementation of (4.1) is how to discretize the following term:

$$
T_{f}=\left(\boldsymbol{K} \int_{0}^{2 \pi} \boldsymbol{\theta} D_{\theta}^{\alpha-1}\left(\boldsymbol{\theta} \cdot \boldsymbol{w}_{h}\right) M(\theta) d \theta, \boldsymbol{v}_{h}\right)
$$

We assume that $M(\theta)$ has the discrete form

$$
M(\theta)=\sum_{k=1}^{L} \omega_{k} \delta\left(\theta-\theta_{k}\right), \quad \sum_{k=1}^{L} \omega_{k}=1,
$$

where $\delta$ is the Dirac delta function. For brevity, we assume $\boldsymbol{K}=K \boldsymbol{I}$, then

$$
T_{f}=\sum_{k=1}^{L} \omega_{k} K\left(D_{\theta_{k}}^{\alpha-1}\left(\boldsymbol{\theta}_{k} \cdot \boldsymbol{w}_{h}\right), \boldsymbol{\theta}_{k} \cdot \boldsymbol{v}_{h}\right)
$$

Since $\boldsymbol{\theta}_{k}=\left(\cos \theta_{k}, \sin \theta_{k}\right)$ and $\boldsymbol{w}_{h}, \boldsymbol{v}_{h} \in \boldsymbol{H}_{h}$ are piecewise constant vector functions with the basis functions $(0,1)^{T}$ and $(1,0)^{T}$, the key implementation of $T_{f}$ lies in the computation of

$$
\hat{T}_{f}^{\theta}=\left(D_{\theta}^{\alpha-1} \chi_{K^{\prime}}, \chi_{K}\right), \quad K^{\prime}, K \in \mathcal{T}_{h},
$$

where $\chi_{S}$ is an indicator function for a set $S$ in $\mathcal{R}^{2}$.
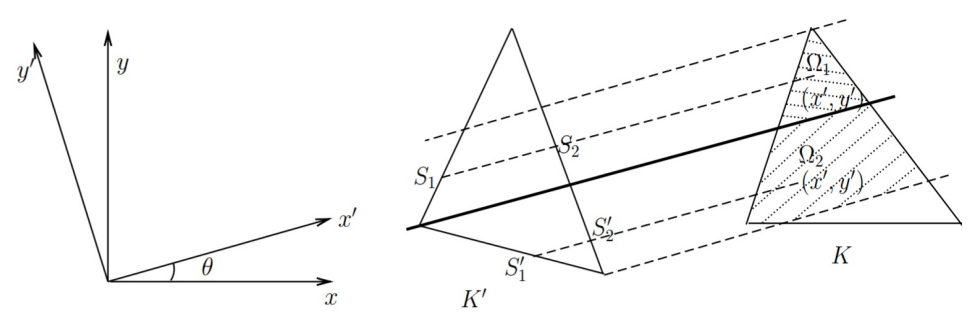

Fig. 1: Illustration for computing $\hat{T}_{f}^{\theta}$ on the unstructured mesh.

If $\mathcal{T}_{h}$ is an unstructured mesh with a triangular partition of $\Omega$, we can compute $\hat{T}_{f}^{\theta}$ as follows: Let $\nu=1-\alpha, \Omega_{1}, \Omega_{2} \in K$, we have

$$
\begin{aligned}
\hat{T}_{f}^{\theta} & =\left(D_{\theta}^{\alpha-1} \chi_{K^{\prime}}, \chi_{K}\right)_{K} \\
& =\int_{\Omega_{1}}\left(\frac{\left(x^{\prime}-S_{1}\right)^{\nu}}{\Gamma(\nu+1)}-\frac{\left(x^{\prime}-S_{2}\right)^{\nu}}{\Gamma(\nu+1)}\right) d x^{\prime} d y^{\prime}+\int_{\Omega_{2}}\left(\frac{\left(x^{\prime}-S_{1}^{\prime}\right)^{\nu}}{\Gamma(\nu+1)}-\frac{\left(x^{\prime}-S_{2}^{\prime}\right)^{\nu}}{\Gamma(\nu+1)}\right) d x^{\prime} d y^{\prime},
\end{aligned}
$$

where $S_{1}, S_{2}, S_{1}^{\prime}, S_{2}^{\prime}$ are the coordinates in the rotating coordinate system (see Figure 1). 

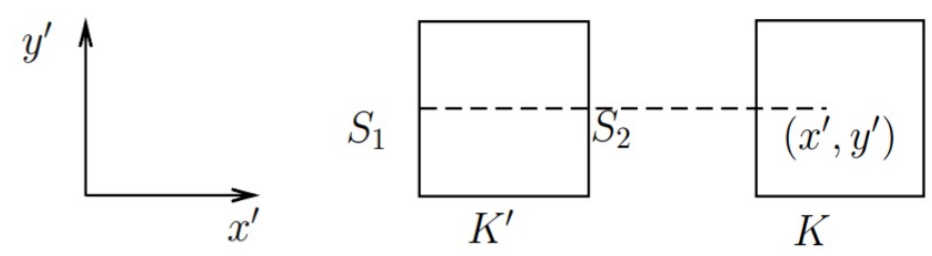

Fig. 2: Illustration for computing $\hat{T}_{f}^{\theta}$ on the structured mesh.

The implementation of $\hat{T}_{f}^{\theta}$ can also be similarly implemented on the structured mesh. For simplicity, we implement (4.1) on the structured mesh with

$$
M(\theta)=\sum_{k=1}^{4} \frac{1}{4} \delta\left(\theta-\theta_{k}\right), \text { where } \theta_{k}=\frac{\pi}{2}(k-1) .
$$

Then, when $\theta=0, \pi / 2, \pi, 3 \pi / 2$, we have (see Figure 2)

$$
\hat{T}_{f}^{\theta}=\left(D_{\theta}^{\alpha-1} \chi_{K^{\prime}}, \chi_{K}\right)_{K}=\int_{K}\left(\frac{\left(x^{\prime}-S_{1}\right)^{\nu}}{\Gamma(\nu+1)}-\frac{\left(x^{\prime}-S_{2}\right)^{\nu}}{\Gamma(\nu+1)}\right) d x^{\prime} d y^{\prime} .
$$

\section{$5 \quad$ Numerical experiments}

In this section we show some numerical results to verify the efficiency of the expanded mixed finite element method. In the following examples, we assume the porous medium is isotropic and $\boldsymbol{K}=\boldsymbol{I}$. We implement the proposed algorithm on the structured mesh with $M(\theta)=\sum_{k=1}^{4} \frac{1}{4} \delta\left(\theta-\theta_{k}\right)$, where $\theta_{k}=\frac{\pi}{2}(k-1)$.

Example 1. In this example we test the steady state space fractional Darcy flow (2.1) on a unit square domain with $81 \times 81$ grid. We let $f=1$ and $f=-1$ in four grid cells of $\mathcal{T}_{h}$ respectively and let $f=0$ in other region.

We choose $\alpha=0.1,0.5,0.7,0.9$ to test the algorithm. From Figure 3 we can clearly see how the parameter $\alpha$ influences the solution.

Example 2. We remark that the expanded mixed FEM can also be applied to solve the following space fractional transport in porous media:

$$
\begin{aligned}
\frac{\partial c}{\partial t}+\nabla \cdot\left(\boldsymbol{u} c+\boldsymbol{D}(\boldsymbol{u}) \nabla_{M}^{\alpha} c\right) & =f & & \text { in } \Omega, \\
c & =0 & & \text { in } \mathcal{R}^{2} \backslash \Omega, \\
c(x, 0) & =c_{0}(x) & & \text { in } \Omega .
\end{aligned}
$$

In this example, we test the space fractional transport on $[0,4] \times[0,1]$ with $128 \times 32$ grid. We assume the velocity $\boldsymbol{u}=(0.1,0)^{T}$. We denote $r^{2}=(x-$ 

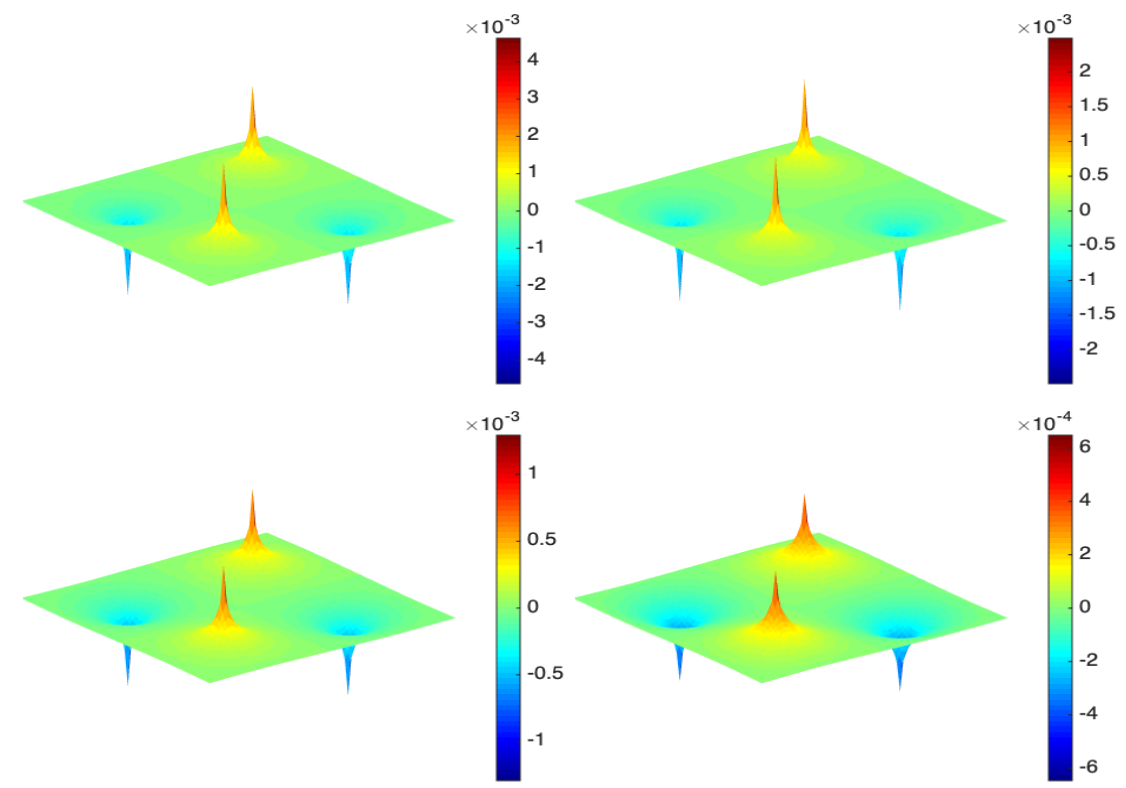

Fig. 3: With $\boldsymbol{K}=1$. Top-Left: solution of pressure $(\alpha=0.1)$. Top-Right: solution of pressure $(\alpha=0.5)$. Bottom-Left: solution of pressure $(\alpha=0.7)$. Bottom-Right: solution of pressure $(\alpha=0.9)$.
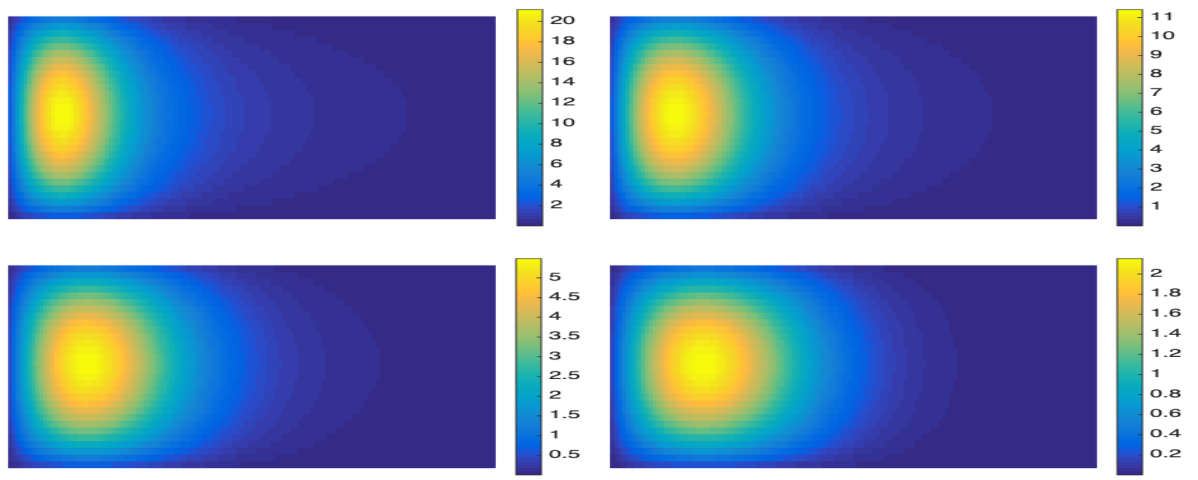

Fig. 4: The concentration solutions with different $\alpha$ at $T=1.2$ with time step $\delta t=0.01$. Top-Left: $\alpha=0.1$. Top-Right: $\alpha=0.5$. Bottom-Left: $\alpha=0.7$. Bottom-Right: $\alpha=0.9$. 
$1 / 8)^{2}+(y-1 / 2)^{2}$ and let $f=0, c_{0}(\boldsymbol{x})=10^{5} \times 2^{1-1 /\left(1-5 r^{2} / 4\right)}$ in the local region $r^{2} \leq 10^{-3}$ and $c_{0}(\boldsymbol{x})=0$ in other region.

We test this example by choosing $\alpha=0.1,0.5,0.7,0.9$ and compute the solutions with time step $\delta t=0.01$ until the time $T=1.2$. From Figure 4 we can also clearly see how the parameter $\alpha$ influences the distribution of concentration at a fixed time. We can see that the diffusion effect is more obvious when the parameter $\alpha$ becomes large.

\section{Conclusion}

In this paper we discuss an expanded mixed finite element method for the solution of the two dimensional space fractional Darcy flow in porous media. The locally mass-conservation can be retained by this mixed scheme. The wellposedness of the expanded mixed formulation is proved and the implementation of the algorithm is given in details. Numerical results are shown to verify the efficiency of this mixed scheme. The mixed scheme for the space fractional Darcy flow with non-homogeneous boundary conditions will be investigated in the future work.

\section{References}

1. R. Adams, Sobolev Spaces, Academic Press, New York, 1975.

2. W. Bu, Y. Tang, J. Yang, Galerkin finite element method for two-dimensional Riesz space fractional diffusion equations, J. Comput. Phys., 276 (2014), pp. 26-38.

3. M. Caputo, Models of flux in porous media with memory, Water Resour. Res., 36 (2000), pp. 693-705.

4. H. Chen and H. Wang, Numerical simulation for conservative fractional diffusion equations by an expanded mixed formulation, J. Comput. Appl. Math., 296 (2016), pp. $480-498$.

5. V. J. Ervin, J. P. Roop, Variational formulation for the stationary fractional advection dispersion equation, Numer. Methods Partial Differential Equations, 22 (2005), pp. $558-576$.

6. V. J. Ervin, N. Heuer, J. P. Roop, Numerical approximation of a time dependent, nonlinear, space-fractional diffusion equation, SIAM J. Numer. Anal. 45 (2007), pp. $572-591$.

7. V. J. Ervin, J. P. Roop, Variational solution of fractional advection dispersion equations on bounded domains in $R^{d}$, Numer. Methods Partial Differential Equations, 23 (2007), pp. 256-281.

8. V. J. Ervin, N. Heuer, J. P. Roop, Regularity of the solution to 1-D fractional order diffusion equations, Math. Comp., 87 (2018), pp. 2273-2294.

9. G. Gao and Z. Sun, A compact finite difference scheme for the fractional subdiffusion equations, J. Comput. Phys., 230 (2011), pp. 586-595.

10. J. H. He, Approximate analytical solution for seepage flow with fractional derivatives in porous media, Comput. Methods Appl. Mech. Engrg., 167 (1998), pp. 57-68.

11. B. Jin, R. Lazarov, J. Pasciak, W. Rundell, Variational formulation of problems involving fractional order differential operators, Math. Comp., 84 (2015), pp. 26652700 . 
12. C. Li, F. Zeng, F. Liu, Spectral approximations to the fractional integral and derivative, Fract. Calc. Appl. Anal., 15 (2012), pp. 383-406.

13. A. Lischke, G. Pang, M. Gulian, F. Song, C. Glusa, X. Zheng, Z. Mao, W. Cai, M. M. Meerschaert, M. Ainsworth, G. Em Karniadakis, What is the fractional Laplacian? A comparative review with new results, J. Comput. Phys., 404 (2020), 109009.

14. F. Liu, P. Zhuang, I. Turner, K. Burrage, V. Anh, A new fractional finite volume method for solving the fractional diffusion equation, Applied Mathematical Modelling, 38 (2014), pp. 3871-3878.

15. M.M. Meerschaert, C. Tadjeran, Finite difference approximations for fractional advection-dispersion flow equations, J. Comput. Appl. Math., 172 (2004), pp.6577.

16. M. M. Meerschaert, H. P. Scheffler, C. Tadjeran, Finite difference methods for two dimensional fractional dispersion equation, J. Comput. Phys., 211 (2006), pp. 249-261.

17. M. M. Meerschaert, J. Mortensen, S. W. Wheatcraft, Fractional vector calculus for fractional advection-dispersion, Physica A, 367 (2006), pp. 181-190.

18. J. P. Roop, Computational aspects of FEM approximation of fractional advection dispersion equations on bounded domains in $R^{2}$, J. Comput. Appl. Math., 193 (2006), pp. 243-268.

19. H. Wang, T. S. Basu, A fast finite difference method for two-dimensional spacefractional diffusion equations, SIAM J. Sci. Comput. 34 (2012), pp. 2444-2458. 